\title{
Entre ville et montagne : habitat, peuplement et terroirs dans les Pyrénées de l'Est de l'Antiquité tardive au Moyen Âge (Cerdagne, $\mathrm{III}^{\mathrm{e}}-\mathrm{XII}^{\mathrm{e}}$ siècle)
}

Thèse de doctorat en archéologie et en histoire sous la codirection de F. Hautefeuille et $C$. Rendu, université de Toulouse 2 Jean Jaurès, soutenue le 6 novembre 2020

Noémie Luault

\section{(2) OpenEdition}

\section{Édition électronique}

URL : https://journals.openedition.org/cem/18662

DOI : $10.4000 /$ cem. 18662

ISSN : 1954-3093

Éditeur

Centre d'études médiévales Saint-Germain d'Auxerre

\section{Référence électronique}

Noémie Luault, «Entre ville et montagne : habitat, peuplement et terroirs dans les Pyrénées de l'Est de I'Antiquité tardive au Moyen Âge (Cerdagne, \|\|$^{\mathrm{e}}$-x॥" ${ }^{\mathrm{e}}$ siècle) », Bulletin du centre d'études médiévales d'Auxerre | BUCEMA [En ligne], 25.2 | 2021, mis en ligne le 31 décembre 2021, consulté le 22 septembre 2022. URL : http://journals.openedition.org/cem/18662; DOI : https://doi.org/10.4000/cem.18662

Ce document a été généré automatiquement le 22 septembre 2022.

\section{c) (†)}

Creative Commons - Attribution - Pas d'Utilisation Commerciale - Partage dans les Mêmes Conditions 4.0 International - CC BY-NC-SA 4.0

https://creativecommons.org/licenses/by-nc-sa/4.0/ 


\section{Entre ville et montagne : habitat, peuplement et terroirs dans les Pyrénées de l'Est de l'Antiquité tardive au Moyen Âge (Cerdagne, III $^{\mathrm{e}}$-XII ${ }^{\mathrm{e}}$ siècle)}

Thèse de doctorat en archéologie et en histoire sous la codirection de F. Hautefeuille et $C$. Rendu, université de Toulouse 2 Jean Jaurès, soutenue le 6 novembre 2020

Noémie Luault

\section{Problématiques}


1 À l'est des Pyrénées, la Cerdagne se présente sous la forme d'une vaste plaine d'une altitude moyenne de 1200 mètres, encadrée de sommets culminant jusqu'à plus de 2900 mètres. Perché dans la zone axiale de la chaîne, ce bassin intramontagnard s'ouvre vers l'orient et la Méditerranée via la vallée de la Têt. À l'ouest, le cours du Sègre le relie à la Péninsule ibérique. Aujourd'hui partagé entre France et Espagne, ce territoire voit s'implanter, au I ${ }^{\text {er }}$ siècle av. J.-C., le cheflieu de cité de Iulia Libica, l'actuelle Llívia (fig. 1 à 3).

Fig. 1 - Localisation de la Cerdagne à l'est des Pyrénées (DAO N. Luault).
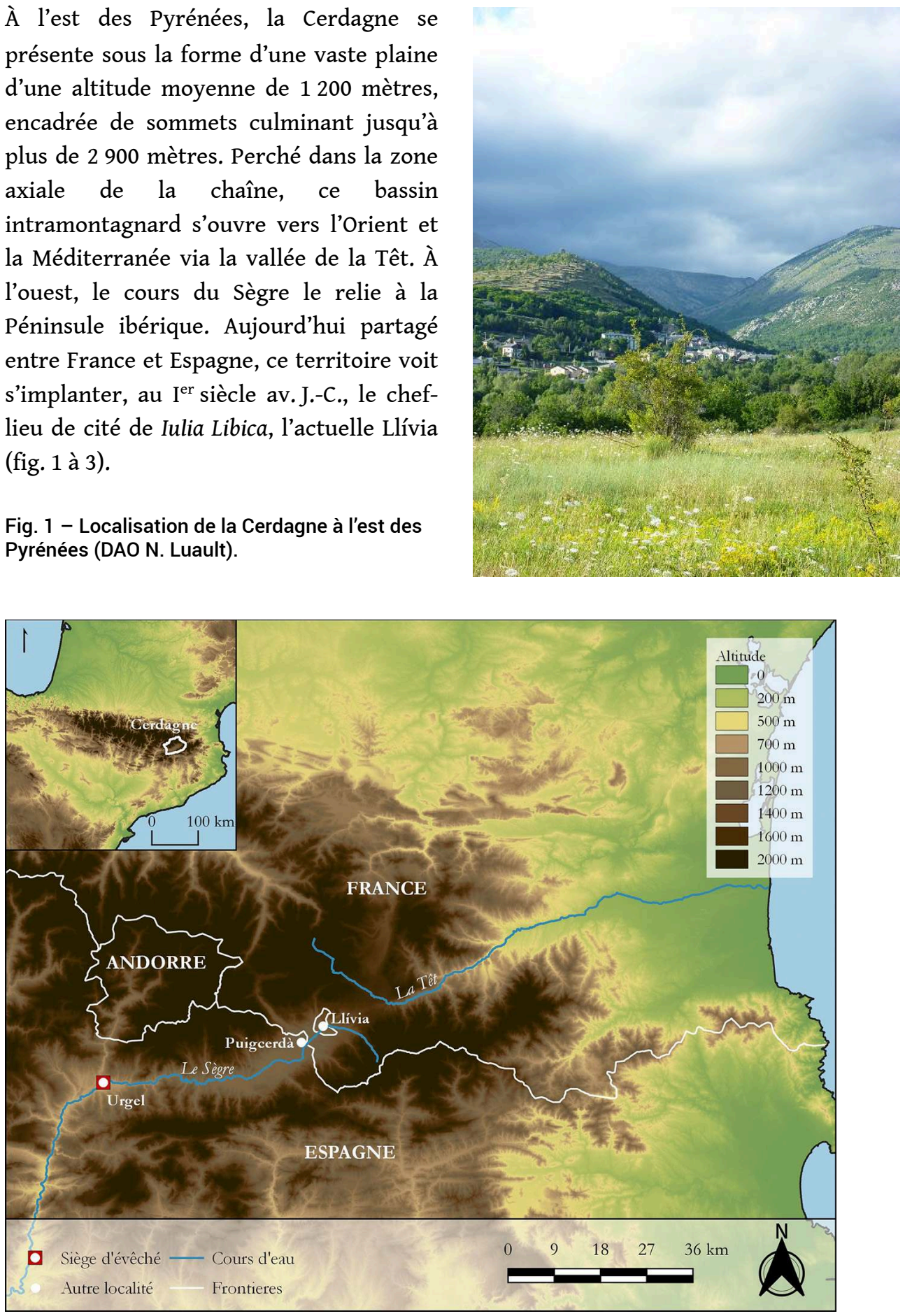
Fig. 2 - Localisation de Llívia et des sites évoqués dans le texte (DAO N. Luault).

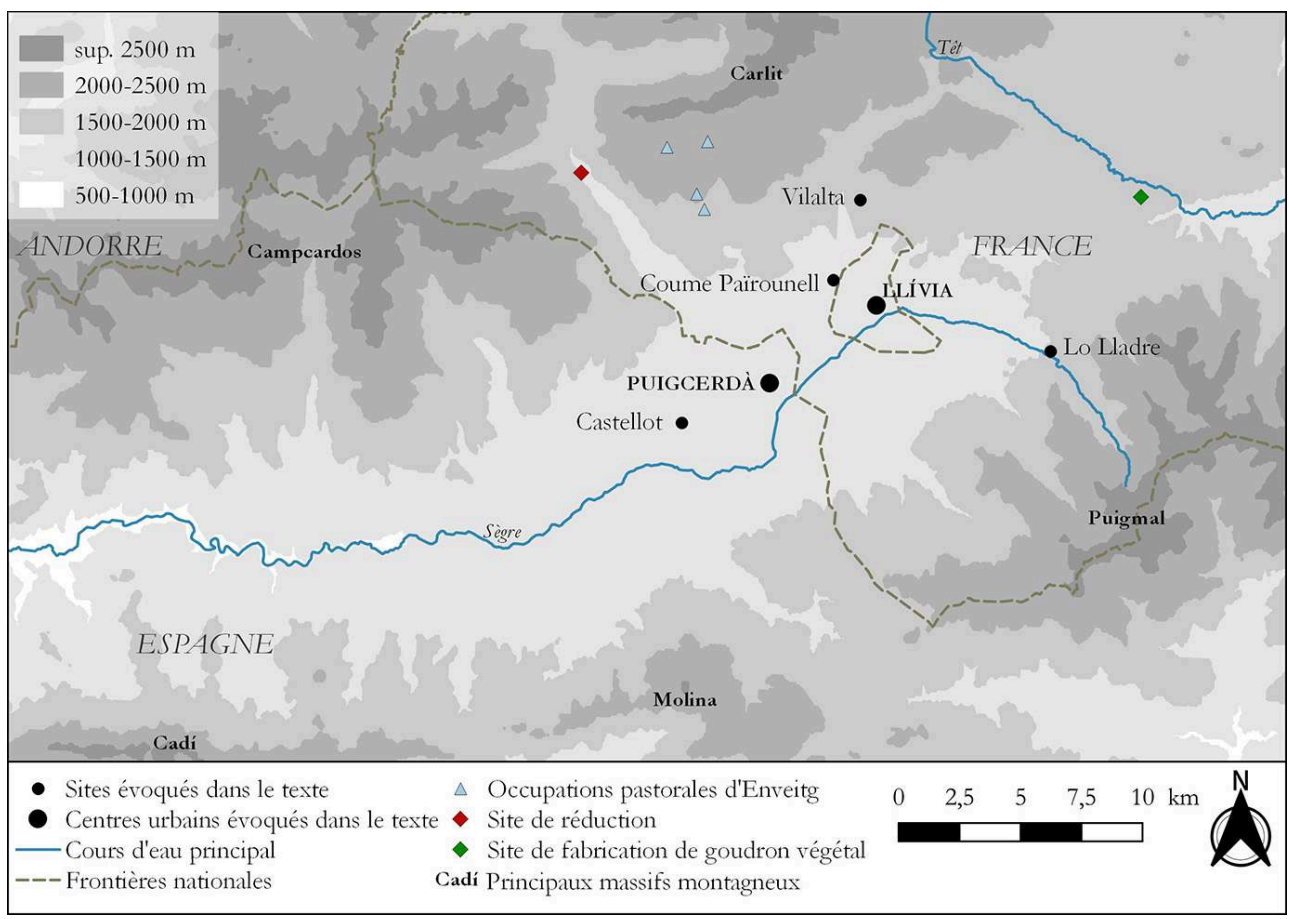

Fig. 3 - Vue de la colline surplombant Llívia depuis le nord-est. À l'arrière-plan, vue de la plaine de Cerdagne, des massifs du Cadi et de La Molina (cl. N. Luault).

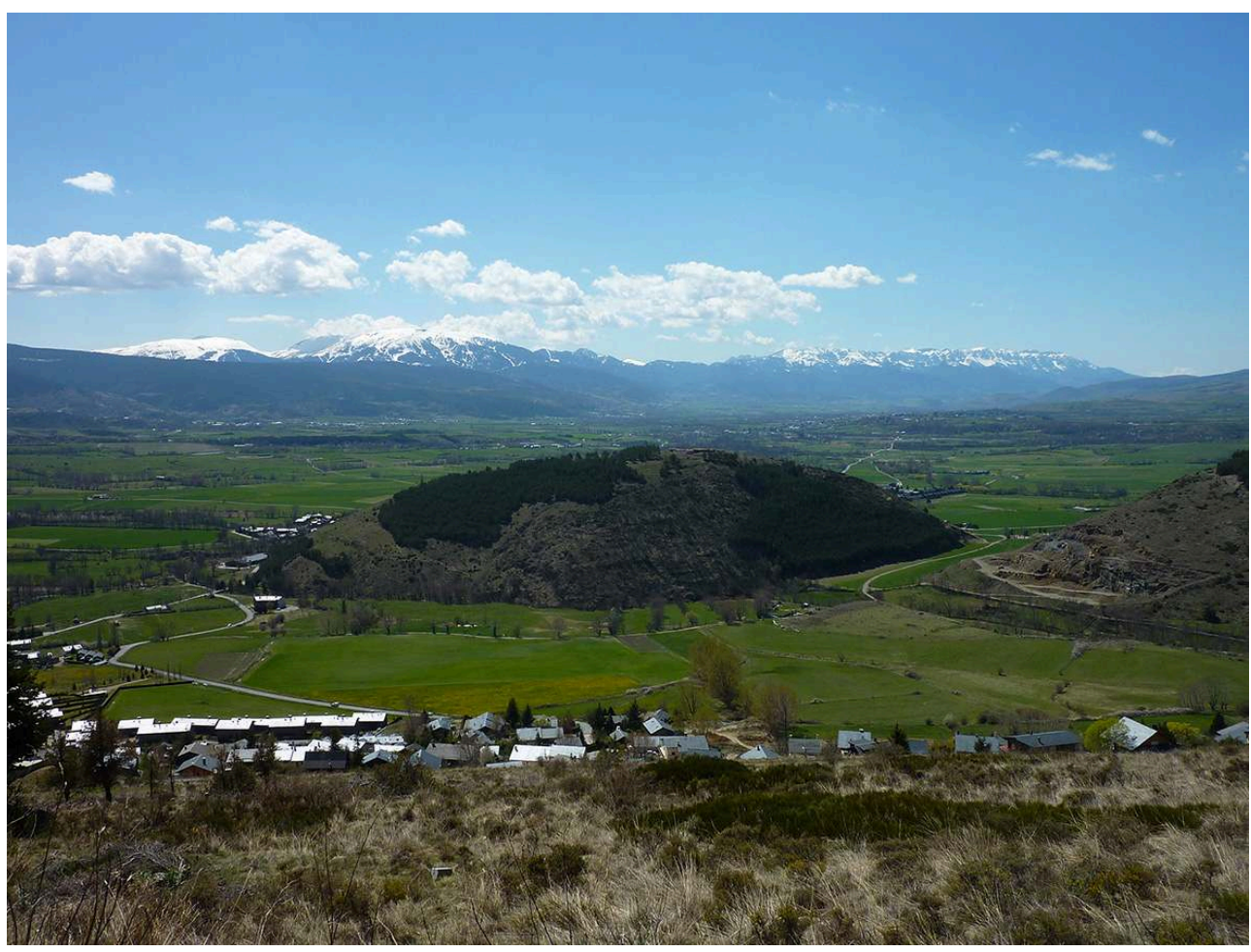

2 Localisée le long d'une ramification de la Via Domitia, en marge de la province de Tarraconaise, Llívia perd probablement son statut de chef-lieu de cité à la fin de l'Antiquité. Lors du second concile de Tolède en 531, en effet, c'est Urgel, une localité située une quarantaine de kilomètres à l'ouest de Llívia, qui est désignée comme siège 
épiscopal ${ }^{1}$. Au siècle suivant, dans la chronique de Julien de Tolède relatant, en 672 , l'expédition du roi wisigoth Wamba, la mention de Llívia comme un simple castrum confirme qu'elle n'a pas le rang de cité épiscopale ${ }^{2}$. L'emploi de ce terme renvoie cependant à des fonctions militaires et politico-administratives, signalant que l'ancienne Iulia Libica parvient à conserver malgré tout une certaine importance. De fait, au moins à partir du IX ${ }^{e}$ siècle, le chef-lieu déclassé coiffe un comté au sein de la cité épiscopale d'Urgel. Ce n'est qu'à une période déjà avancée du Moyen Âge, à partir $\mathrm{du} \mathrm{XI} \mathrm{I}^{\mathrm{e}}$ siècle, que son rôle politique s'essouffle définitivement au profit du site d'Hix et de son palais comtal, flanqués d'une fortification implantée sur une hauteur voisine. Le rôle prépondérant de ce nouveau pôle est définitivement entériné avant les années 1170 , moment où le roi Alphonse $\mathrm{I}^{\mathrm{er}}$ ordonne le transfert d'Hix sur l'éminence toute proche, fondant ainsi Puigcerdà ${ }^{3}$.

Ce travail de recherche s'est intéressé aux dynamiques de l'habitat, du peuplement et des terroirs entre le $\mathrm{III}^{\mathrm{e}}$ et le XII $\mathrm{XI}^{\mathrm{e}}$ siècle, période bornée à l'amont par le déclassement du chef-lieu de cité et à l'aval par la création de Puigcerdà. Llívia, de ses évolutions dans la géographie politico-administrative régionale à ses transformations intra-urbaines, a constitué un premier point d'ancrage. Seul exemple de chef-lieu de cité implanté au cœur du massif des Pyrénées, elle possède néanmoins une particularité : elle se situe à l'est de la chaîne, dans un environnement montagnard.

4 Formant un second point d'ancrage, la montagne constitue un terrain neuf dans le champ de la recherche sur les systèmes de peuplement de l'Antiquité tardive et du haut Moyen Âge. Si des travaux de plus en plus nombreux ont en effet éclairé, durant les deux dernières décennies, les transformations au long cours des espaces agro-sylvopastoraux de haute montagne, force est de constater qu'entre ces deux extrémités du peuplement que constituent, d'un côté la ville, de l'autre le semis des habitats saisonniers d'altitude, la relation n'est pas encore établie. C'est elle qui a constitué, véritablement, notre objet de recherche. Sans chercher à définir de nouvelles méthodes, il s'est agi d'appliquer des outils ailleurs éprouvés à un espace qui, d'un point de vue archéologique, n'avait pas encore, en montagne, droit de cité : celui de l'habitat permanent et de ses terroirs, en considérant que mutations de la trame du peuplement et dynamiques agraires pouvaient avoir, ici aussi, leur importance.

\section{Démarche adoptée}

5 Au cœur de la plaine d'altitude, cette enquête a démarré à Llívia même, avec un bilan des connaissances archéologiques concernant le centre urbain, partagé entre une agglomération basse (environ 1200 mètres) et une colline la surplombant - Puig de Llívia, 1350 mètres $^{4}$. Nos pas nous ont conduits ensuite sur le site de La Coume Païrounell - Angoustrine-Villeneuve-des-Escaldes, fig. 4, 5 et 6 -, correspondant à un habitat groupé permanent occupé entre le début $d u v^{e}$ et la charnière des $I^{e}$ et $\mathrm{x}^{\mathrm{e}}$ siècles. 
Fig. 4 - Localisation du site de La Coume Païrounell dans la périphérie de Llívia et à l'entrée de la vallée d'Angoustrine, principale voie d'accès vers les espaces d'altitude du massif du Carlit - axe représenté par une flèche noire - (DAO N. Luault).

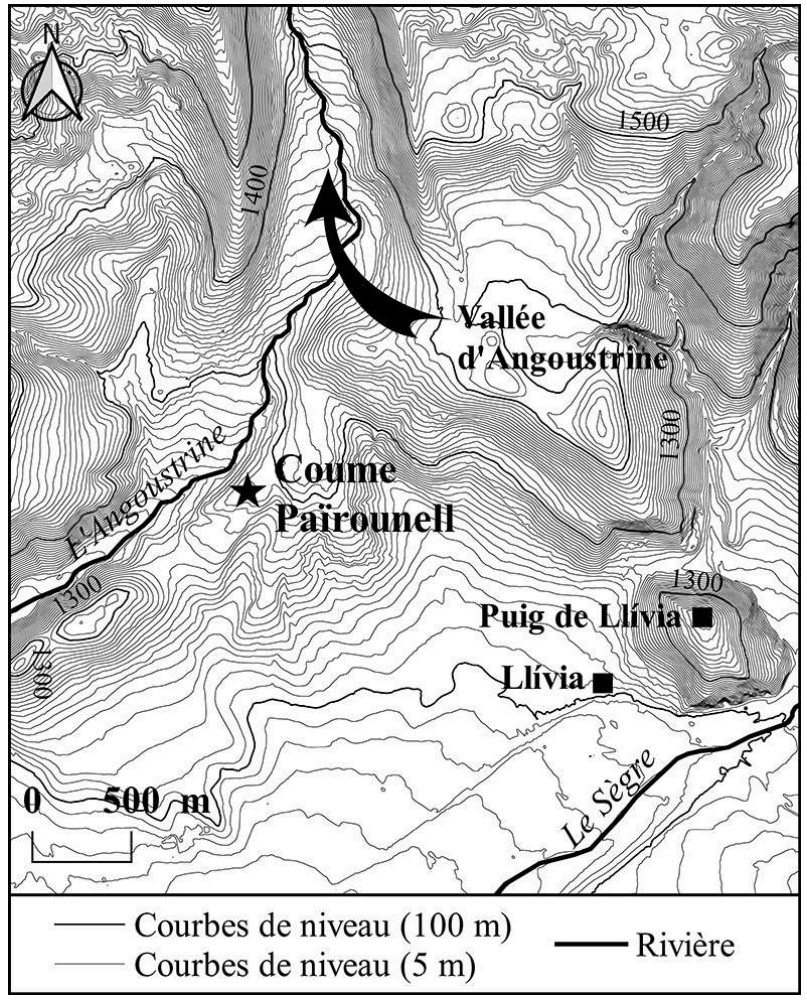

Fig. 5 - Plan du site de La Coume Païrounell à l'issue de la campagne de fouilles de 2017 (DAO N. Luault).

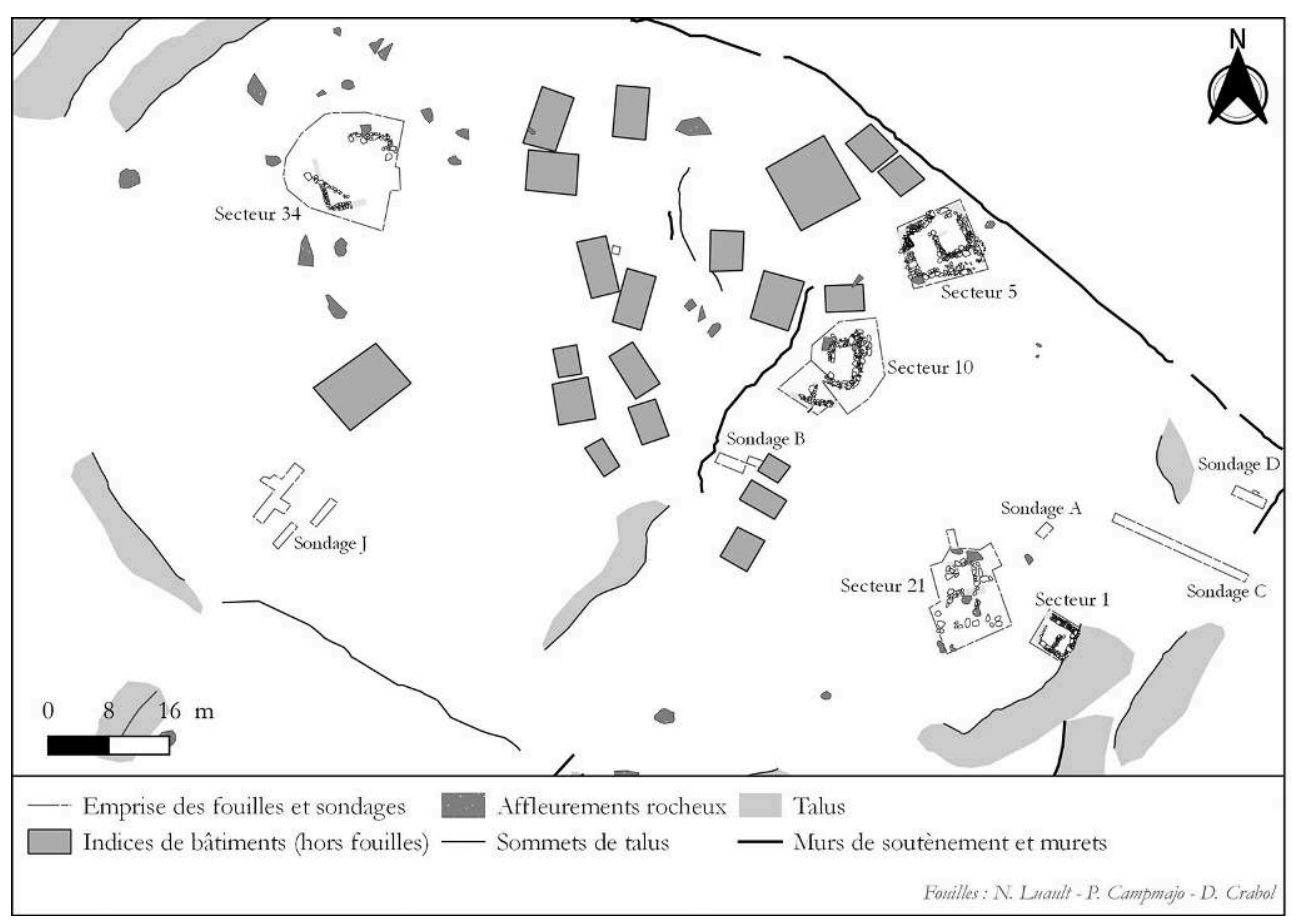


Fig. 6 - Fouilles en cours du secteur 34 sur le site de La Coume Païrounell - 2017, deux bâtiments des $\mathrm{V}^{\mathrm{e}}$ et $\mathrm{Vl}^{\mathrm{e}}$-VII ${ }^{\mathrm{e}}$ siècles - (cl. N. Luault).

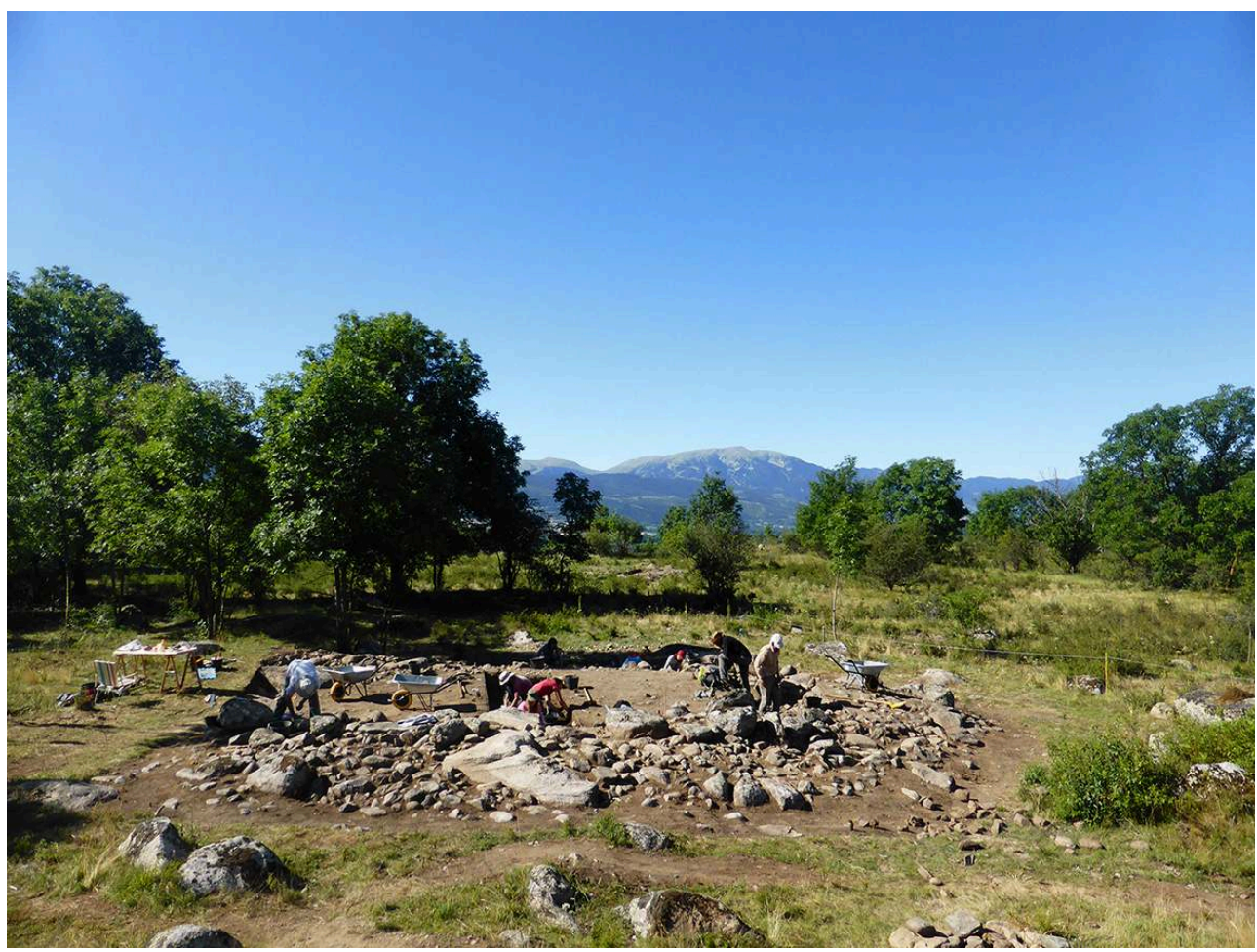

Depuis cet établissement de hauteur implanté à moins de deux kilomètres de l'ancienne Iulia Libica, il s'agissait d'éclairer les rythmes du centre urbain par sa périphérie. Ce site avait bénéficié d'une première approche dans les années 2000 , sous la forme de fouilles exploratoires et d'un relevé topographique partiel ${ }^{5}$. À ces résultats préliminaires se sont ajoutées les données recueillies au cours de quatre années d'investigations archéologiques conduites entre 2014 et $2017^{6}$. Plusieurs approches ont été mises en place: une analyse de surface - relevés au GPS différentiel et prospections géophysiques $^{7}-$, des fouilles et sondages ciblés, et des études des artefacts et écofacts conduites avec la collaboration de différents spécialistes ${ }^{8}$.

$7 \quad$ L'analyse du peuplement s'est ensuite étendue à l'échelle du bassin valléen (1000 km²) en associant deux démarches. En premier lieu, il s'agissait de comparer les trajectoires des quelques sites documentés par l'archéologie, révélés par les fouilles programmées, puis les interventions de plus en plus nombreuses d'archéologie préventive réalisées ces deux dernières décennies de part et d'autre de la frontière. Trois sites ont ainsi fait l'objet d'une synthèse plus détaillée (fig. 2) : celui de Lo Lladre (Llo), un habitat perché protohistorique probablement réoccupé aux $\mathrm{V}^{\mathrm{e}}$ et $\mathrm{VI}^{\mathrm{e}}$ siècles, puis entre les $\mathrm{x}^{\mathrm{e}}$ et $\mathrm{XII}^{\mathrm{e}}$ siècles $^{9}$; celui du Castellot (Bolvir), également un site de hauteur protohistorique réinvesti des environs de l'an Mil jusqu'au milieu du $\mathrm{XII}^{\mathrm{e}}$ siècle $^{10}$; et enfin celui de Vilalta (Targasonne), un habitat groupé implanté à près de 1700 mètres d'altitude, qui se développe à partir du XI ${ }^{\mathrm{e}}$ siècle et jusqu'au XIV ${ }^{\mathrm{e}}$ siècle $^{11}$.

8 En second lieu, les dynamiques perçues ont été reliées à celles dévoilées par les sources écrites pour les $\mathrm{IX}^{\mathrm{e}}$-XII ${ }^{\mathrm{e}}$ siècles. En constituant une base de données spatialisée, fondée sur un échantillon de près de deux cents chartes provenant des fonds de la cathédrale d'Urgel ${ }^{12}$, le parti d'une approche quantitative et spatialisée des sources a été adopté, 
afin d'aborder les transformations des structures territoriales et d'encadrement, à plusieurs niveaux ${ }^{13}$.

9 C'est enfin à l'échelle des quelques communes qui entourent Llívia $\left(100 \mathrm{~km}^{2}\right)$ que nous avons cherché à saisir, entre la ville et ses montagnes, les dynamiques des terroirs agro-sylvo-pastoraux. Les rythmes d'exploitation des espaces agraires de la plaine d'altitude ont été documentés à travers deux types de prospections pédestres, en champs labourés (fig. 7) et en prairies. Leurs résultats ont ensuite été confrontés à ceux acquis en haute montagne ${ }^{14}$.

Fig. 7 - Prospections en cours dans les secteurs de labours de la plaine de Cerdagne - 2015, parcelle au sud de Llívia - (cl. N. Luault).

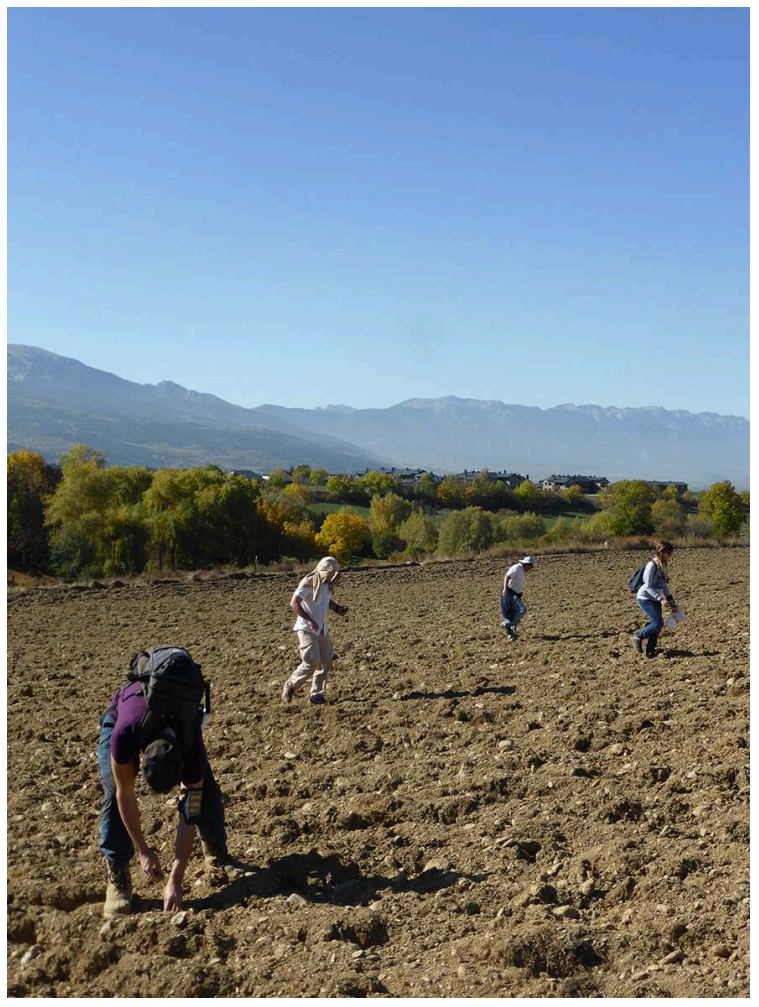

\section{Principaux résultats}

10 Au début de la période étudiée, aborder Llívia par sa périphérie a permis d'appréhender sous un nouvel angle les restructurations de l'ancien chef-lieu de cité. Fondée au $\mathrm{I}^{\mathrm{er}}$ siècle av. J.-C., Iulia Libica montre des signes d'essoufflement dès le courant du II siècle : le forum semble perdre son caractère public et certains espaces d'habitat sont abandonnés. Au cours des deux siècles qui suivent, l'agglomération est caractérisée par un dynamisme très faible. Ce n'est qu'aux $\mathrm{v}^{\mathrm{e}}$ et $\mathrm{VI}^{\mathrm{e}}$ siècles qu'une réoccupation plus intensive est documentée. De nouveaux espaces d'habitat sont ainsi créés dans la zone nord-est du forum. Dans ce secteur, la découverte d'un singe, inhumé avec des éléments de parure militaire, témoignerait de la présence temporaire d'une armée à Llívia. Dans le même temps, sur la colline, l'existence d'une fortification est à envisager.

11 C'est dans cet intervalle qu'émerge l'établissement de hauteur de La Coume Païrounell, à seulement deux kilomètres de l'ancienne Iulia Libica. Implanté dans la partie la plus 
haute d'un promontoire culminant à près de 1300 mètres d'altitude, ce site occupe une superficie d'environ un hectare. Après une première phase d'occupation à la fin de l'âge du bronze ou au début de l'âge $\mathrm{du}$ fer ${ }^{15}$, le gisement est réoccupé à partir du début du $\mathrm{V}^{\mathrm{e}}$ siècle. Dès cette période, à côté d'activités agricoles et domestiques, la métallurgie du fer semble constituer un artisanat de prédilection. Deux étapes de la chaîne opératoire sont documentées jusqu'au milieu du $\mathrm{vl}^{\mathrm{e}}$ siècle: la forge mais aussi l'affinage, une opération postérieure à la réduction du minerai, mais précédant le travail de forge proprement dit. Témoin d'un développement de l'habitat perché aux $\mathrm{v}^{\mathrm{e}}$ et $\mathrm{vI}^{\mathrm{e}}$ siècles, dont le site Lo Lladre constitue un second exemple, l'investissement de ce site d'habitat permet d'envisager la Llívia de cette période comme un site multipolaire. Implantée au débouché de la vallée d'Angoustrine (fig. 4 et fig. 8), La Coume Païrounell apparaît comme une sorte d'excroissance de Llívia, en position idoine pour accéder aux ressources d'altitude du massif du Carlit, et en particulier aux ressources en bois, combustible indispensable aux activités métallurgiques pratiquées sur le site.

Fig. 8 - Vue du massif du Carlit et de la vallée d'Angoustrine depuis le site de La Coume Païrounell (cl. N. Luault).

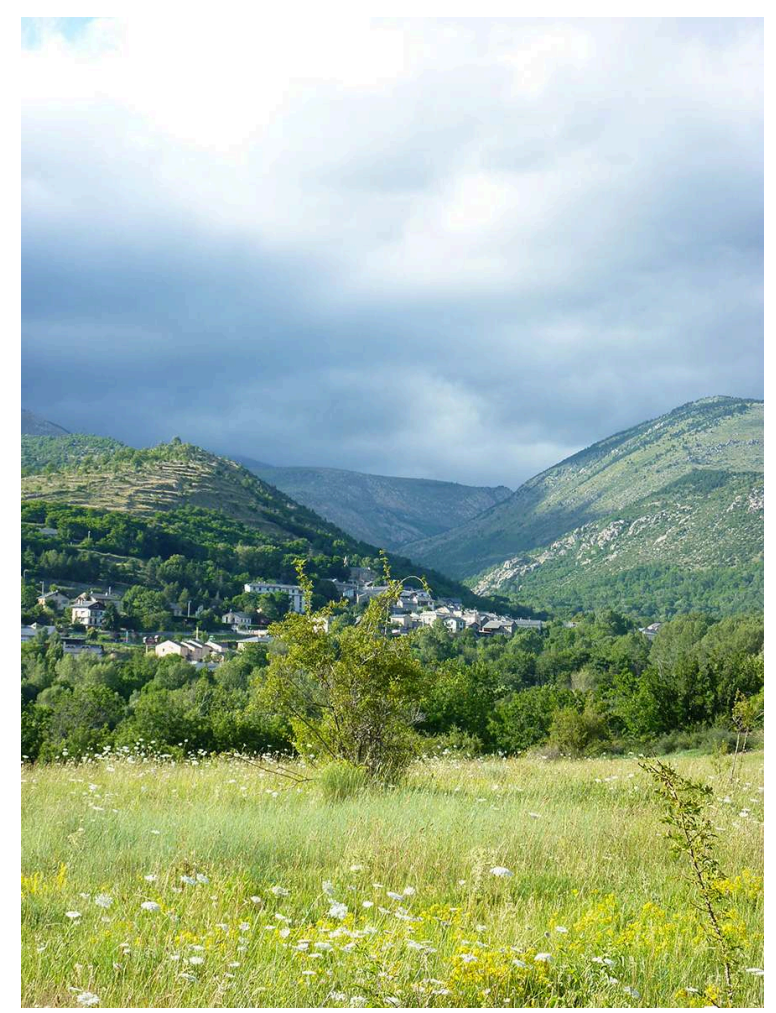

Or, aux cours des $\mathrm{v}^{\mathrm{e}}$ et $\mathrm{vI}^{\mathrm{e}}$ siècles, les versants semblent s'orienter vers une exploitation des ressources forestières, en lien avec des activités artisanales. Alors que les données polliniques de la montagne d'Enveitg montrent un recul assez marqué du pin et du sapin à partir $d u v^{e}$ siècle $^{16}$, deux découvertes récentes nous orientent dans cette direction. La première correspond à un four destiné à la fabrication de goudron végétal mis au jour sur la commune de Bolquère, à 1620 mètres d'altitude, et daté des $\mathrm{v}^{\mathrm{e}}$ et $\mathrm{VI}^{\mathrm{e}}$ siècles $^{17}$. La seconde découverte se situe dans la vallée du Carol, à 1430 mètres d'altitude. Il s'agit d'un site de réduction daté entre la fin du $\mathrm{IV}^{\mathrm{e}}$ et le début du $\mathrm{VI}^{\mathrm{e}}$ siècle ${ }^{18}$. 
13 Si l'on peine encore à mieux cerner les évolutions des $\mathrm{VII}^{\mathrm{e}}$ et $\mathrm{VIII}^{\mathrm{e}}$ siècles, plusieurs éléments ont été mis en évidence. Sur le site de La Coume Païrounell, on observe une continuité d'occupation. Succédant aux premiers temps de l'habitat, une première série de remaniements a probablement lieu entre le milieu du $\mathrm{vI}^{\mathrm{e}}$ et le milieu du $\mathrm{VII}^{\mathrm{e}}$ siècle. On note ainsi une réorientation fonctionnelle du secteur 21 , de la métallurgie du fer vers des activités domestiques et agricoles ou encore la construction d'un nouvel édifice dans le secteur 34 (fig. 5 et 6). Pour l'heure, nous n'avons pas documenté d'activités métallurgiques durant cet intervalle d'environ un siècle. Ces dernières sont attestées de nouveau à partir du milieu du viI ${ }^{\mathrm{e}}$ siècle et jusqu'au tournant des $\mathrm{IX}^{\mathrm{e}}$ $\mathrm{x}^{\mathrm{e}}$ siècles, moment où le site semble abandonné. Durant cette dernière phase d'occupation, l'artisanat du fer semble désormais se limiter à la forge et cohabite toujours avec des activités agricoles et domestiques. Dans le même temps, sur le versant sud du Carlit, les indices palynologiques et archéologiques d'une pression agropastorale plus marquée se multiplient. Dans un système complémentaire avec l'élevage et la sylviculture, les activités agricoles pourraient à ce moment acquérir une certaine importance dans l'économie des versants. En témoigneraient les indices d'une culture locale de céréales à près de 1900 mètres d'altitude ${ }^{19}$ ou encore, plus bas sur la montagne, une première mise en terrasses du terroir de Vilalta (1 650 mètres) ${ }^{20}$.

Dans les derniers temps de la période étudiée (IX ${ }^{\mathrm{e}}-\mathrm{XII}^{\mathrm{e}}$ siècle), la confrontation des données archéologiques et textuelles fournit de nouvelles clefs de lecture du territoire au moment où Llívia s'éclipse progressivement. Chef-lieu comtal au moins à partir du $\mathrm{IX}^{\mathrm{e}}$ siècle, l'ancienne cité ne semble plus désignée à la fin $\mathrm{du} \mathrm{x}^{\mathrm{e}}$ siècle que comme le siège d'un pagus, correspondant à une circonscription intermédiaire, visiblement centrée sur le castrum de Llívia. Cette période correspond à l'apparition dans les textes de fortifications qui semblent, comme Llívia, s'inscrire dans la continuité de pôles hérités de l'Antiquité tardive et s'avèrent tenues par le comte de Cerdagne. C'est le cas notamment du site de Lo Lladre, où de nouvelles phases d'occupation sont justement documentées à partir $\mathrm{du} \mathrm{x}^{\mathrm{e}}$ siècle.

Passé l'an Mil, le comte semble progressivement se désintéresser de l'ancien chef-lieu de cité. Au nord de Llívia, les possessions comtales sont progressivement cédées. Dans ces secteurs caractérisés par une forte présence publique, l'abandon du site de La Coume Païrounell, avant le milieu du $\mathrm{x}^{\mathrm{e}}$ siècle, pourrait constituer un prélude à ce mouvement. Mais c'est surtout au regard des recompositions du peuplement aux environs de l'an Mil que cet abandon mérite d'être considéré. Ce phénomène semble en effet coïncider avec l'apparition dans les textes de Villeneuve, un village situé à près de 500 mètres du site de La Coume Païrounell et destiné à devenir le siège d'une paroisse. Il fait écho à la naissance, à plus de 1700 mètres d'altitude, du site de Vilalta. Formé au $\mathrm{XI}^{\mathrm{e}}$ siècle, ce village abritant une église paroissiale au XII ${ }^{\mathrm{e}}$ siècle semble correspondre à une forme neuve d'habitat groupé dont l'éclosion pourrait traduire une nouvelle organisation économique et sociale. Dans le même temps, sa localisation à la lisière des estives invite, à nouveau, à s'intéresser aux évolutions des terroirs de montagne. Cette période semble en effet correspondre à une phase de forte pression agro-pastorale, dont l'apogée se situe au XIII ${ }^{\mathrm{e}}$ siècle.

16 Au terme de cette enquête, la Cerdagne - un espace perché au cœur du massif des Pyrénées et en marge de la province de Tarraconaise - semble s'inscrire pleinement dans les rythmes du peuplement de la Gaule méditerranéenne. On peut se demander dans quelle mesure l'implantation de Llívia au cœur de ce territoire a pu contribuer au 
dynamisme de cette région montagnarde. À n'en pas douter, les espaces étudiés ont bénéficié de plusieurs atouts: situés sur un itinéraire majeur reliant, à l'est des Pyrénées, le sud de la Gaule au nord-est de la Péninsule ibérique, ils offrent par ailleurs une configuration particulière, permettant l'exploitation de ressources variées. Pour aller plus loin et évaluer dans quelle mesure les trajectoires de ce territoire sont singulières, les évolutions mises en évidence sont appelées, dans les années à venir, à être confrontés aux évolutions de l'habitat, du peuplement et des terroirs d'autres régions pyrénéennes et, plus largement, montagnardes.

Reçu : 16 juin 2021 - Accepté : 17 décembre 2021

\section{NOTES}

1. C. GASCóN, «El bisbe Just i els orígens de la diòcesi d'Urgell », Urgellia, 19 (2015-2018), p. 411-438.

2. « Castrum Libiae, quod est Cirritaniae caput », JULIEN DE TOLÈDE, Histoire du roi Wamba, éd. W. LEVISON, Clermont-Ferrand, 2011, p. 10-11.

3. Cartas de población y franquicia de Cataluña, éd. J. M. FONT I RIUS, Madrid/Barcelone, 1969, n 154.

4. Ce bilan s'appuie notamment sur la thèse récente de J. GUÀRDIA, El forrum romà de Iulia Libica $i$ l'arqueologia urbana de Llivia (Cerdanya), thèse de doctorat, Universitat Autònoma de Barcelona, Barcelone, 2018.

5. P. CAMPMAjo, D. CRABOL, E. BILle, C. RAynAud, M.-P. RUAS, G. PARENT et C. RENDU, «Un atelier de traitement du fer sur le site du haut Moyen Âge de La Coume Païrounell à Angoustrine (PyrénéesOrientales): premiers résultats ", in A. CATAFAU (dir.), Activités, échanges et peuplement entre Antiquité et Moyen Âge en Pyrénées-Orientales et Aude, Perpignan, 2007, p. 137-163 ; P. СамPMAJO, D. CRABOL, C.RENDU, G.PARENT ET M.-P.RUAS, «Angoustrine-Villeneuve-les-Escaldes. Sondages et prospection. », Bulletin de l'AAPO, 20 (2005), p. 15-16. ; P. CAMPMAJO, D. CRABOL, G. PARENT, C. RAYNAUD, C. RENDU ET M.P. RUAS, «Angoustrine-Villeneuve-les-Escaldes: La Coume Païrounell. Fouilles ", Bulletin de l'AAPO, 19 (2004), p. 16-19.

6. N. LUAULT (dir.), La Coume Païrounell. Rapports de fouilles programmées 2014 à 2017, DRAC/SRA Languedoc-Roussillon/Occitanie, Montpellier, 2014 à 2016 et 2020, 4 vol.

7. Prospections au géoradar et magnétomètre effectuées par T. Gragson (University of Georgia, Department of Anthropology).

8. Études de mobilier réalisées avec les collaborations de C. Raynaud (UMR 5140 ASM), J. Kotarba (Inrap) et P. Campmajo (GRAHC) pour la céramique et le verre; G. Pagès (UMR 7041 ArScAn) pour la paléométallurgie; A. Casenove et $C$. Respaut (Inrap) pour le matériel lithique; études archéobotaniques menées, pour la carpologie, par M.-P. Ruas (UMR 7209 AASPE) et C. Hallavant (UMR 5608 TRACES) ; pour l'anthracologie, par V. Py (UMR 5602 GEODE).

9. P. CAMPMAJo (dir.) Llo1. Rapports de fouilles programmées 2011 à 2013, DRAC/SRA LanguedocRoussillon, Montpellier, 2011 à 2013, 3 vol.

10. A. CUBO, O. MERCADAL, J. MORERA, O. OLESTI et J. OLLER, «El Castellot de Bolvir. Un vilatge cerdà dels segles XI-XII ", in V Congrés d'Arqueologia Medieval i Moderna a Catalunya (Barcelona, 22-25 de maig de 2014), Barcelone, 2015, p. 687-700. 
11. O. PASSARRIUS, C. CALASTRENC et C. RENDU (dir.), Targasonne - Thémis/Vilalta: rapport final de diagnostic, DRAC/SRA Languedoc-Roussillon/Pôle archéologique départemental 66, Montpellier/ Perpignan, 2009.

12. «Els documents de l'Arxiu Capitular de la Seu d'Urgell», éd. C.BARAUT, Urgellia, II-XI, 1979-1992.

13. Ce travail s'appuie également sur les analyses d'É. Bille (É. BILLE, Seigneurs, maisons et vacants : la Cerdagne du dixième au quatorzième siècle, thèse de doctorat, université Toulouse - Jean Jaurès, Toulouse, 2004).

14. Concernant la haute montagne, les principales données auxquelles nous faisons appel proviennent des travaux interdisciplinaires conduits sur la commune d'Enveitg (fig. 2), sous la direction de C. Rendu: C. RENDU, La montagne d'Enveig: une estive pyrénéenne dans la longue durée, Canet, 2003 ; EAD. (dir.), PCR: Estivage et structuration sociale d'un espace montagnard, la Cerdagne, rapports intermédiaires 2003 à 2005, DRAC/SRA Languedoc-Roussillon, Montpellier, 2003 à 2005, 3 vol.

15. Découverts à l'occasion d'un sondage (sondage J, fig. 5) effectué au cours de la dernière campagne réalisée sur le site, les vestiges liés à cette première implantation devront faire l'objet d'une fouille plus étendue afin de mieux la caractériser.

16. D. GALOP, diagramme inédit.

17. A. TOLEDO (dir.), Bolquère - La Serra de les Artigues centre technique d'exploitation routière. Un atelier de production de goudron végétal (ou station de résinier) de l'Antiquité tardive. Rapport final d'opération. Diagnostic archéologique, DRAC/SRA Occitanie/Inrap Méditerranée, Montpellier/Nîmes, 2018. Une fouille préventive, dont le rapport est en cours, a été réalisée par la suite sous la direction de Jérôme Kotarba (Inrap Méditerranée).

18. G. PAGÈs (dir.), Rapport final de prospection thématique triennale (2015-2017). Rapport de sondage archéologique: les productions de fer antiques et médiévales dans le massif du Canigou (66), DRAC/SRA Occitanie, Montpellier, 2017.

19. M.-P. RUAS, C. RENDU, A. BERGERET et A. CATAFAU, « Glanes et cultures en haute montagne d'après les restes de graines et de fruits carbonisés de deux sites médiévaux de Cerdagne et du Capcir (Pyrénées-Orientales) », in Les ressources naturelles des Pyrénées du Moyen Âge à l'époque moderne: exploitation, gestion, appropriation, Perpignan, 2005, p. 147-184.

20. C. rendu， O. passarrius， C. calastrenc， R. Julia， M. llubes， P. illes， P. campmajo，C. Jodry, D. CRABOL, E. BILLE, M. CONESA, D. BOUSQUET et V. LALLEMAND, « Reconstructing past terrace fields in the Pyrenees insights into land management and settlement from the Bronze Age to the Early Modern era at Vilalta (1650 masl, Cerdagne, France) », Journal of Field Archaeology, 40-4 (2015), p. 461-480.

\section{INDEX}

Keywords : mountain, town, habitat, settlement, agro-sylvo-pastoral land, rural territories, Late Antiquity, Middle Ages

Mots-clés : montagne, ville, habitat, peuplement, terroirs agro-sylvo-pastoraux, territoires ruraux, Antiquité tardive, Moyen Âge

Index géographique : Pyrénées, Cerdagne, Catalogne 
AUTEUR

NOÉMIE LUAULT

Docteure de l'UMR 5608 Traces ; responsable d'opération chez Hadès 